\title{
Ertapenem: Una nueva clase de carbapenem
}

\author{
RICARDO MORALES I. ${ }^{1}$
}

\section{Ertapenem}

Ertapenem is an new carbapenem that, compared to imipenem and meropenem, has a prolonged half life that allows its administration once daily and may be prescribed in severe infections treated as outpatients. Its antimicrobial spectrum is directed mainly against enteric Gram negative rods including betalactamase extended spectrum producers but its antibacterial activity against Pseudomonas spp or Acinetobacter spp is scarce. Besides ertapenem is active against Haemophilus influenzae y Streptococcus pneumoniae .including penicillin resistant strains and non Bacteroides strict anaerobes. It has no bactericidal activity against Enterococcus spp. Because of its favorable spectrum and safety profile ertapenem is well indicated in mixed infections as abdominal or pelvic sepsis, severe soft tissue infections, complexed urinary tract infections and community acquired pneumonia with suspicion of a mixed etiology.

Key words: Ertapenem; Spectrum; Safety; Clinical efficacy.

Los carbapenems son antimicrobianos $\beta$ lactámicos de amplio espectro de actividad antimicrobiana. Existían a la fecha dos carbapenems: imipenem/cilastatina y meropenem de uso principalmente en infecciones intrahospitalarias y de múltiples dosis diarias. En noviembre del 2001 la FDA y en abril del 2002 la Unión Europea aprobaron la incorporación al uso clínico de ertapenem (MK-0826) de la investigación de Merck Sharp \& Dohme, un nuevo carbapenem con algunas diferencias a las otras moléculas.

Es así, como recientemente se ha propuesto una nueva clasificación de los carbapenems ${ }^{1}$, dado que no son compuestos homogéneos, tienen espectro antimicrobiano y características farmacocinéticas diferentes:

Grupo 1: incluye carbapenems de amplio espectro, con limitada actividad contra bacilos Gram negativos no fermentadores, que están indicados principalmente en infecciones adquiridas en la comunidad. Ejemplo: ertapenem.

Grupo 2: incluye carbapenems de amplio espectro, activos contra bacilos Gram negativos no fermentadores (Acinetobacter spp, Pseudomonas spp), indicados principalmente en infecciones nosocomiales.
Ejemplo: imipenem/cilastatina y meropenem.

Grupo 3: incluye carbapenems con actividad contra Staphylococcus spp meticilina resistente (aún no licenciados).

\section{Estructura química de ertapenem}

Ertapenem es un $1-\beta$-metil carbapenem, estable a las dihidropeptidasas renales por lo que no requiere coadministración de cilastatina como inhibidor, que tiene un grupo hidroxietilo en el carbono 6, similar a imipenem y meropenem, confiriéndole estabilidad frente a hidrólisis por $\beta$ lactamasas.

A diferencia de otros carbapenems, ertapenem posee un grupo metilo en el carbono 4, una cadena sulfhidrilo y una cadena lateral aniónica benzoato que modifica la carga molecular global aumentando la unión a proteínas plasmáticas y alargando su vida media ${ }^{2}$ (Figura 1).

\section{Mecanismo de acción}

Ertapenem se une a las proteínas ligadoras de penicilina bloqueando la síntesis de pared bacteriana celular. Presenta fuerte unión a PBP 1a, 1b, 


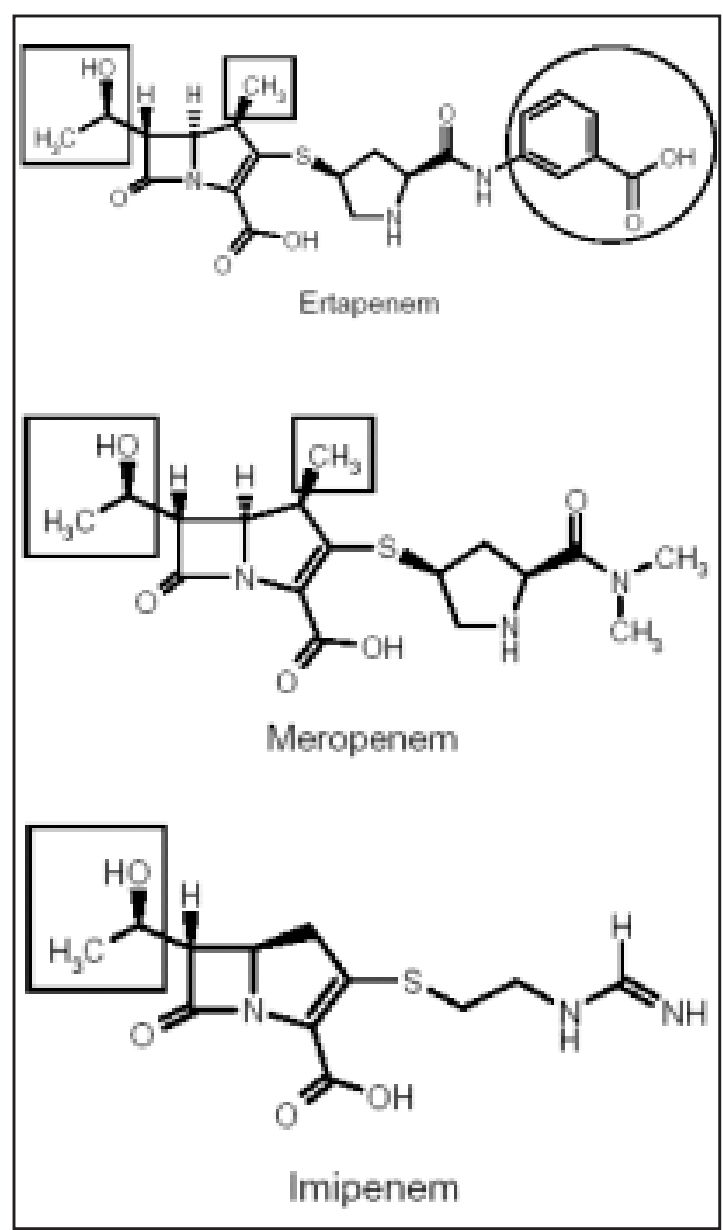

Figura 1. Estructura química de carbapenems ${ }^{1}$.

2, 3, 4 y 5 mostrando mayor afinidad por PBP 2 y PBP 3. Su acción es bactericida y tiene efecto postantibiótico contra cocáceas Gram positivas ${ }^{2,3}$.

\section{Farmacocinética}

Tiene una alta unión a proteínas plasmáticas $>95 \%$ (reversible) con una vida media de 4,9 \pm 0,7 horas lo que permite dosificación una vez al día en administración endovenosa o intramuscular.

La depuración total es 10 a $18 \%$ mayor que la filtración glomerular sugiriendo una posible vía secretoria de eliminación.

Ertapenem reconstituido con lidocaína $1 \%$ es absorbido prácticamente en su totalidad luego de su administración intramuscular, con una biodisponibilidad promedio de $90 \%$. El pico de concentración plasmática seguido de 1 gr intramuscular se alcanza aproximadamente a las 2,3 horas ${ }^{2}$. (Tabla 1).

En adultos jóvenes sanos luego de $1 \mathrm{gr}$ ev se recupera $80 \%$ en la orina y $10 \%$ en las heces. El metabolito principal es un derivado inactivo en forma de anillo abierto formado por la hidrólisis del anillo $\beta$-lactámico ${ }^{4}$.

Las concentraciones plasmáticas medias de ertapenem a las 12 horas luego de $1 \mathrm{gr}$ ev fueron más del doble del punto de corte de susceptibilidad de $4 \mu \mathrm{g} / \mathrm{ml}$.

Las concentraciones urinarias medias de ertapenem estuvieron por encima del punto de corte de susceptibilidad de $4 \mu \mathrm{g} / \mathrm{ml}^{5}$.

La concentración de ertapenem en lesiones cutáneas ampollares es mayor de $4 \mu \mathrm{g} / \mathrm{ml}$ (CIM a la que el $90 \%$ de bacterias aisladas son eliminadas).

La concentración en leche materna es baja y no se detecta luego de 5 días de suspensión del tratamiento en mujeres con infecciones pélvicas ${ }^{2,6}$.

\section{Ertapenem en insuficiencia renal y hepática}

La vida media de ertapenem se mantiene en 4,5 hrs en insuficiencia renal leve, 6,1 horas en moderada, 10,6 en avanzada y 14,1 horas en insuficiencia renal terminal. La depuración por hemodiálisis es de 30\% (Tabla 2).

No ha sido establecido ajuste de dosis en insuficiencia hepática (considerar que la recuperación es sólo de $10 \%$ en las heces).

\section{Farmacodinamia}

Ertapenem posee propiedades farmacodinámicas similares a otros carbapenems: una acción bactericida rápida y no dependiente de la concentración en bacilos Gram negativos.

Las CIM de ertapenem para bacilos Gram negativos, excepto Acinetobacter spp y Pseudo-

Tabla 1. Farmacocinética de administración endovenosa e intramuscular.

Concentraciones plasmáticas de ertapenem después de administración de dosis única

\begin{tabular}{|c|c|c|}
\hline \multirow[t]{2}{*}{ Tiempo } & \multicolumn{2}{|c|}{$\begin{array}{c}\text { Promedio de concentraciones } \\
\text { plasmáticas }\end{array}$} \\
\hline & $1 \mathrm{~g} \mathrm{IV}^{*}$ & $1 \mathrm{~g} \mathrm{IM}$ \\
\hline $0,5 \mathrm{~h}$ & $155 \mu \mathrm{g} / \mathrm{ml}$ & $33 \mu \mathrm{g} / \mathrm{ml}$ \\
\hline 1 & $115 \mu \mathrm{g} / \mathrm{ml}$ & $53 \mu \mathrm{g} / \mathrm{ml}$ \\
\hline $2 \mathrm{~h}$ & $83 \mu \mathrm{g} / \mathrm{ml}$ & $67 \mu \mathrm{g} / \mathrm{ml}$ \\
\hline $4 \mathrm{~h}$ & $48 \mu \mathrm{g} / \mathrm{ml}$ & $57 \mu \mathrm{g} / \mathrm{ml}$ \\
\hline $6 \mathrm{~h}$ & $31 \mu \mathrm{g} / \mathrm{ml}$ & $40 \mu \mathrm{g} / \mathrm{ml}$ \\
\hline $8 \mathrm{~h}$ & $20 \mu \mathrm{g} / \mathrm{ml}$ & $27 \mu \mathrm{g} / \mathrm{ml}$ \\
\hline $12 \mathrm{~h}$ & $9 \mu \mathrm{g} / \mathrm{ml}$ & $13 \mu \mathrm{g} / \mathrm{ml}$ \\
\hline $18 \mathrm{~h}$ & $3 \mu \mathrm{g} / \mathrm{ml}$ & $4 \mu \mathrm{g} / \mathrm{ml}$ \\
\hline $24 \mathrm{~h}$ & $1 \mu \mathrm{g} / \mathrm{ml}$ & $2 \mu \mathrm{g} / \mathrm{ml}$ \\
\hline
\end{tabular}

- Infusión constante en 30 minutos $^{1}$. Cunha B. Drugs of Today 200238 (3): 195-213 (adaptado). 
Tabla 2. Dosificación en insuficiencia renal (adaptado de ${ }^{1}$ )

\begin{tabular}{ll}
\hline Clearance de creatinina & Dosis \\
\hline$>60 \mathrm{ml} / \mathrm{min}$ & $1 \mathrm{gr}$ \\
$30-60 \mathrm{ml} / \mathrm{min}$ & $1 \mathrm{gr}$ \\
$<30 \mathrm{ml} / \mathrm{min}$ & $500 \mathrm{mg}$ \\
\hline
\end{tabular}

Suplemento de $150 \mathrm{mg}$ post diálisis, si esta ocurre dentro de las 6 horas de administrada la dosis.

monas spp, son menores que las de imipenem y significativamente más bajas que las de ceftriaxona, excepto para $H$. influenzae ${ }^{2}$.

\section{Microbiología}

La actividad in vitro de ertapenem ante más de 5.550 aislados provenientes de Norteamérica y más de 3.450 cepas de Europa y Australia se comparó con imipenem, cefepime, ceftriaxona y piperacilina/tazobactam.

Ertapenem fue el más activo contra enterobacterias con CIM $90 \leq 1 \mu \mathrm{g} / \mathrm{ml}^{7}$.

La CIM $_{90}$ de ertapenem para Streptococcus spp, Staphylococcus spp susceptibles a meticilina $\mathrm{y}$ anaerobios fue $\leq 0,5 \mu \mathrm{g} / \mathrm{ml}$.

La actividad de ertapenem contra Streptococcus pneumoniae se analizó en 3.328 cepas, las CIM varían entre 0,015 y $0,5 \mu \mathrm{g} / \mathrm{ml}$ (181 cepas eran resistentes a penicilina). El 100\% de las cepas fue susceptibles 8 .
Para $H$. influenzae (331 cepas) la actividad de ertapenem no se modifica ante la presencia de $\beta$ lactamasas. La $\mathrm{CIM}_{90}$ fue de $0,06 \mu \mathrm{g} / \mathrm{ml}$ con rango de $\leq 0,015$ a $0,12 \mu \mathrm{g} / \mathrm{ml}^{8}$.

Las bacterias menos susceptibles a ertapenem fueron Acinetobacter spp y Pseudomonas aeruginosa con CIM mayores a $16 \mu \mathrm{g} / \mathrm{ml}$ por lo que no debe usarse en infecciones por estos patógenos y por lo tanto, con precaución en infecciones nosocomiales donde estos patógenos pueden ser prevalentes.

La mayoría de las cepas de Enterococcus faecalis fueron resistentes ${ }^{2}$ por lo que también se desaconseja su uso clínico en infecciones por este patógeno.

Se comparó actividad in vitro contra 556 cepas clínicas anaerobias.

Ertapenem inhibe el 99,1\% de todos los aislados a $4 \mu \mathrm{g} / \mathrm{ml}$, comparable a imipenem, meropenem, piperacilina/tazobactam y metronidazol.

Todos los aislados de Prevotella, Porphyromonas, Fusobacterium y Peptostreptococccus fueron susceptibles a ertapenem.

Cinco cepas de Bacteroides fragilis mostraron susceptibilidad reducida a ertapenem CIM $\geq 8 \mu \mathrm{g} / \mathrm{ml}^{9}$ (Tabla 3 ).

\section{Actividad de ertapenem contra enterobacterias productoras de $\beta$-lactamasas}

Ertapenem es relativamente estable frente a $\beta$-lactamasas AMPc y $\beta$-lactamasas de espectro extendido (BLEE) y tiene una buena actividad

Tabla 3. Puntos de corte para susceptibilidad a ertapenem ${ }^{1}$

\begin{tabular}{|c|c|}
\hline Interpretación CIM ( $\mu \mathrm{g} / \mathrm{ml})$ & Interpretación diámetro (mm) \\
\hline \multicolumn{2}{|c|}{ Para Enterobacteriaceae y Staphylococcus spp.: } \\
\hline $\begin{array}{l}<2,0 \text { susceptible }(\mathrm{S}) \\
4,0 \text { intermedio }(\mathrm{I}) \\
>8,0 \text { resistente }(\mathrm{R})\end{array}$ & $\begin{array}{l}>19 \text { susceptible }(\mathrm{S}) \\
16-18 \text { intermedio }(\mathrm{I}) \\
<15 \text { resistente }(\mathrm{R})\end{array}$ \\
\hline \multicolumn{2}{|l|}{ Para Haemophilus spp.: } \\
\hline$<0,5$ susceptible $(\mathrm{S})$ & $>19$ susceptible $(\mathrm{S})$ \\
\hline \multicolumn{2}{|l|}{ Para Streptococcus pneumoniae: } \\
\hline$<1,0$ susceptible $(\mathrm{S})$ & $>19$ susceptible $(\mathrm{S})$ \\
\hline \multicolumn{2}{|c|}{ Para Streptococcus spp. distintos a S. pneumoniae: } \\
\hline$<1,0$ susceptible $(\mathrm{S})$ & $>19$ susceptible $(\mathrm{S})$ \\
\hline \multicolumn{2}{|l|}{ Para anaerobios estrictos: } \\
\hline $\begin{array}{l}<4,0 \text { susceptible }(\mathrm{S}) \\
8,0 \text { intermedio }(\mathrm{I}) \\
>16,0 \text { resistente }(\mathrm{R})\end{array}$ & No disponible \\
\hline
\end{tabular}


frente a enterobacterias que producen $\beta$-lactamasas potentes (no carbapenemasas) con CIM entre 0,03 a $0,12 \mu \mathrm{g} / \mathrm{ml}$ y siempre menor a $4 \mu \mathrm{g} / \mathrm{ml}$.

Ertapenem fue menos activo contra cepas productoras de BLEE y de AMPc que las no productoras, pero aún así retiene actividad completa contra estas mismas cepas ${ }^{10,11}$.

Un reporte reciente de 142 cepas clínicas de enterobacterias productoras de $\beta$-lactamasas de espectro ampliado e hiperproductoras de AmpC informa $\mathrm{CIM}_{90}$ menores de $0,03 \mu \mathrm{g} / \mathrm{ml}$ y 0,03 $0,25 \mu \mathrm{g} / \mathrm{ml}$ respectivamente ${ }^{12}$.

Estos hechos, sumado a la dosificación de una vez al día lo presentan como la mejor alternativa terapéutica frente a infecciones producidas por bacterias productoras de BLEE.

\section{Efecto inóculo}

Con un inóculo estándar 1X, la mayoría de las cepas fueron susceptibles a ceftriaxona, cefepime y ertapenem.

Cuando se elevó el inóculo a 10X aumentando la cantidad de $\beta$-lactamasas, muchas de las cepas productoras de BLEE se hicieron muy resistentes con CIM mayor de $128 \mu \mathrm{g} / \mathrm{ml}$ a los antimicrobianos no carbapenémicos.

Las CIM para ertapenem fueron más elevadas pero no alcanzaron concentraciones consideradas como resistencia ${ }^{10}$.

\section{Mecanismos de resistencia a ertapenem}

En un estudio de 455 cepas de $K$. pneumoniae productoras de BLEE aisladas en hemocultivos, la $\mathrm{CIM}_{50}$ para ertapenem fue de $0,094 \mu \mathrm{g} / \mathrm{ml}$.

Se encontraron 5 pacientes con $K$. pneumoniae productoras de BLEE con CIM $\geq 16 \mu \mathrm{g} / \mathrm{ml}$.

Se identificaron BLEE: CTX- M- 2 (6 cepas), SHV-5 (1 cepa) y TEM-10 y SHV-5 (1 cepa).

En otros reportes la resistencia de K. pneumoniae a ertapenem requiere BLEE plasmidial o $\beta$ lactamasas AMPc más una o más mutaciones cromosomales que pueden ser seleccionadas espontáneamente a baja frecuencia ${ }^{10,13}$.

En 20 cepas de $P$. aeruginosas ertapenem no indujo actividad de $\beta$-lactamasas AMPc, a diferencia de meropenem y en menor grado imipenem, probablemente por falta de inducción pero puede reflejar también actividad inhibitoria de $\beta$-lactamasas ${ }^{14}$.

Así, la resistencia a bacilos Gram negativos se relaciona a la combinación de menor acumulación del fármaco en el sitio de acción más hidrólisis en el espacio periplásmico por $\beta$-lactamasas. Aunque existe el potencial riesgo de resistencia cru- zada con imipenem y meropenem, estudios in vitro y resultados de estudios de vigilancia indican que la resistencia cruzada no es completa. En particular, la resistencia de ertapenem para bacilos Gram negativos es inhabitual y la perspectiva histórica aportada por los otros carbapenems sugiere que es poco probable el aumento de la resistencia ${ }^{1,15}$.

Como enfoques de prevención de aparición de resistencia, ertapenem puede usarse por corto tiempo dentro del hospital, seguido de cambio a antimicrobianos orales apropiados o continuar con tratamiento domiciliario dadas las ventajas de dosificación una vez al día y la opción de uso intramuscular.

\section{Eficacia clínica de ertapenem}

Diversos estudios clínicos avalan el uso de ertapenem en:

- Infecciones intra-abdominales complicadas.

- Infecciones complicadas de piel y tejidos blandos.

- Infecciones urinarias complicadas.

- Infecciones pélvicas agudas.

- Neumonía adquirida en la comunidad.

Infecciones intraabdominales complicadas: existen dos estudios clínicos relevantes que comparan ertapenem con piperacilina/tazobactam o con la asociación de ceftriaxona y metronidazol ${ }^{16,17}$.

En el estudio comparativo ertapenem $1 \mathrm{gr}$ ev día (n: 203) versus piperacilina/tazobactam 3,375 gr cada 6 horas ev (n: 193) randomizado doble ciego: las tasas de curación clínica microbiológica a las 4-6 semanas (test de cura) asociado a la cirugía correspondiente fueron $87 \%$ para ertapenem y $81 \%$ para piperacilina/tazobactam ${ }^{16}$.

Infecciones quirúrgicas con aislamiento de Enterococcus spp: se analiza el pronóstico en pacientes tratados con ertapenem versus piperacilina/tazobactam; análisis que incluye a 1.558 pacientes tratados en tres estudios randomizados doble ciego por infecciones intraabdominales complicadas, infecciones pélvicas e infecciones complicadas de piel y tejidos blandos: 223 pacientes tenían cultivos iniciales positivos a Enterococcus spp (20\% de las infecciones intra-abdominales).

No existió diferencia en resultado clínico entre los dos esquemas terapéuticos en pacientes con o sin Enterococcus spp. En infecciones intraabdominales y pélvicas las tasas de cura fueron numéricamente mayores en pacientes con 
Enterococcus spp tratados con ertapenem que con piperacilina/tazobactam ${ }^{18}$.

Infecciones complicadas de piel y tejidos blandos: estudio comparativo ertapenem $1 \mathrm{gr} \mathrm{ev}$ día (n: 185) con piperacilina/tazobactam 3,375 gr ev cada 6 horas ev (n: 174). El diagnóstico más común fue absceso de piel o tejidos blandos y pie diabético infectado. La eficacia analizada como test de curación a los 10 a 21 días después del tratamiento fue $82,4 \%$ para ertapenem y $84,4 \%$ para piperacilina/tazobactam. No hubo diferencias significativas según diagnóstico y severidad de la infección. Un $40 \%$ de las infecciones fueron polimicrobianas en ambos grupos, aproximadamente $18 \%$ tenía más de una especie anaerobia aislada y $S$. aureus fue el patógeno aislado más frecuente (aproximadamente $40 \%$ en ambos grupos). La erradicación bacteriana al momento del test de curación fue de $82,6 \%$ para ertapenem y $83,4 \%$ para piperacilina/tazobactam. La frecuencia y severidad de los eventos adversos relacionados a los fármacos fue similar en ambos gru$\operatorname{pos}^{19}$.

Infecciones urinarias complicadas: un estudio comparativo de ertapenem 1 gr ev día (n: 162) con ceftriaxona 1 gr ev día (n: 176) randomizado doble ciego con switch a ciprofloxacina oral según evolución. Aproximadamente $95 \%$ de los pacientes usaron tratamiento oral en cada grupo. Los patógenos más comúnmente aislados fueron Escherichia coli y Klebsiella pneumoniae. Aproximadamente el 50\% de los pacientes evaluables en ambos grupos tenían infección severa. En el test de curación 5 a 9 días postratamiento, 91,8\% de los pacientes con ertapenem y $93,0 \%$ de los que recibieron ceftriaxona tuvieron respuesta microbiológica favorable independiente de diagnósticos y de severidad. El perfil de seguridad fue similar para ambos medicamentos ${ }^{20}$.

Neumonía adquirida en la comunidad en adultos: estudio comparativo doble ciego de ertapenem 1 gr ev una vez al día (n: 182) con ceftriaxona 1 gr ev día (n: 203). El promedio de duración de terapia ev fue de 4 días para ambos grupos. De los pacientes clínicamente evaluables, $168(92,3 \%)$ en ertapenem y $183(91 \%)$ en ceftriaxona tuvieron respuesta clínica favorable. Streptococcus pneumoniae fue el agente más frecuentemente aislado y se observaron altas tasas de curación para $S$. pneumoniae susceptibles y resistentes a penicilina $(87,5 \%$ y $100 \%$ respectivamente). Ambos tratamientos fueron bien tolerados, los eventos adversos más frecuentes fue- ron diarrea $(2,9 \%$ vs $2,7 \%)$ y náuseas $(0,8 \%$ vs $2,0 \%)$ para ertapenem y ceftriaxona respectivamente $^{21}$.

Infecciones pélvicas agudas (infecciones posparto o cirugías obstétricas e infecciones ginecológicas o postoperatorias): Estudio comparativo doble ciego de ertapenem 1 gr ev (n: 163) con piperacilina/tazobactam 3,375 grs c/6 horas ev (n: 153). El diagnóstico más frecuente fue endomiometritis (75\%). En los patógenos aislados predominaron $E$. coli y especies anaerobias. En la evaluación a las 2 a 4 semanas postratamiento, la respuesta clínica fue de 93,9\% para ertapenem y de $91,5 \%$ para piperacilina/ tazobactam. El perfil de seguridad fue similar en ambos grupos ${ }^{22}$.

\section{Perfil de tolerancia}

En los ensayos clínicos se han incluido aproximadamente 1.954 pacientes (Tabla 4).

La mayoría de los eventos adversos notificados fueron de intensidad leve a moderada, motivando la suspensión del tratamiento en $4,7 \%$ de los pacientes.

El 0,3\% de los pacientes tuvo que suspender el tratamiento por algún efecto adverso de laboratorio.

Se notificaron convulsiones en $0,5 \%$ de los pacientes versus $0,3 \%$ de los tratados con piperacilina/tazobactam y $0 \%$ en los tratados con ceftriaxona.

No se ha establecido eficacia ni seguridad en población pediátrica ${ }^{1}$.

\section{Interacciones medicamentosas}

El ertapenem no inhibe el metabolismo media-

Tabla 4. Efectos adversos clínicos y de parámetros de laboratorio en adultos (n: 1.954)

\begin{tabular}{lr}
\hline Diarrea & $5,5 \%$ \\
Flebitis & $3,7 \%$ \\
Náuseas & $3,1 \%$ \\
Cefalea & $2,2 \%$ \\
Vaginitis & $2,1 \%$ \\
Vómitos & $2,1 \%$ \\
$\uparrow$ ALT & $6 \%$ \\
$\uparrow$ AST & $5,2 \%$ \\
$\uparrow$ F. alcalinas & $3,40 \%$ \\
$\downarrow$ Plaquetas & $2,80 \%$ \\
$\uparrow$ Eosinófilos & $1,10 \%$ \\
\hline
\end{tabular}


do por ninguna de las siguientes isoformas del citocromo P450: 1A2, 2C9, 2C19, 2D6, 2E1 y $3 \mathrm{~A} 4{ }^{1}$.

\section{Conclusiones}

- Ertapenem ofrece cobertura de infecciones complicadas o severas producida por flora mixta de aerobios y anaerobios adquiridas en la comunidad: en casos de infección intraabdominal, infección de piel y tejidos blandos, infección pélvica aguda, disminuyendo la necesidad de dosis múltiples y de asociaciones de antimicrobianos.

- Eficacia demostrada en neumonía comunitaria e infección urinaria complicada.

- Excelente acción contra enterobacterias productoras de BLEE e hiperproductoras de Amp $\mathrm{C}$, constituyendo una importante alternativa a los carbapenems tradicionales en esta indicación.

- Sin actividad frente a Pseudomonas spp y Acinetobacter spp. Pobre actividad frente a Enterococcus spp. Por esto, ertapenem no debe usarse empíricamente en infecciones intrahospitalarias, neumonía asociada a ventilación mecánica o infecciones adquiridas en unidades de cuidados intensivos, debiendo esperar resultados microbiológicos para su uso en estos casos.

- Su farmacocinética y farmacodinámica apoyan la monodosis diaria, fácil de administrar.

- Dosificación sencilla en la disfunción renal.

- Eficacia clínica en pacientes ancianos.

- Sin interacciones medicamentosas.

- Bien tolerado.

- Uso en pacientes ambulatorios con vía de administración intramuscular o endovenosa una vez al día, a diferencia de imipenem y meropenem que requieren múltiples dosis endovenosas, permitiendo un alta hospitalaria precoz en el manejo de infecciones por bacterias multiresistentes.

\section{Resumen}

Ertapenem es un nuevo carbapenem que, comparativamente con imipenem y meropenem, tiene una prolongada vida media que permite su administración una vez al día abriendo la posibilidad a su indicación en infecciones severas en etapa de manejo ambulatorio. Su espectro antimicrobiano está dirigido principalmente hacia bacilos Gram negativos entéricos incluyendo especies productoras de bðlactamasas de espectro extendido pero no tiene bue- na actividad sobre Pseudomonas spp ni Acinetobacter spp. También es activo sobre Haemophilus influenzae y Streptococcus pneumoniae incluyendo cepas resistentes a penicilina y anaerobios estrictos no Bacteroides. Su actividad sobre Enterococcus spp es nula. Por las características de espectro y perfil de seguridad ertapenem es una buena indicación en infecciones de etiología mixta: sepsis abdominal y pélvica, infecciones mayores de tejidos blandos, infecciones urinarias complejas y neumonías adquiridas en la comunidad con sospecha de etiologías mixtas.

\section{Agradecimientos}

Mi reconocimiento al Dr. Guillermo Acuña L. por la lectura crítica del manuscrito y sus valiosas sugerencias.

\section{Bibliografía}

1.- Shah P M, Isaacs R D. Ertapenem, the first of a new carbapenem. J Antimicrob Chemother 2003; 52: 53842.

2.- Cunha B. Ertapenem: a new carbapenem. Drugs Today 2002; 38 (3):195-213.

3.- Odenholt $\mathrm{I}$, Lowdin $\mathrm{E}$, Cars $\mathrm{O}$ et al. In vitro pharmacodynamic studies of L-749,345 in comparison with imipenem and ceftriaxone against Gram-positive and Gram-negative bacteria. Antimicrob Agents Chemother 1998 42: 2365-70.

4.- Majumdar A, Musson D G, Birk K L et al. Pharmacokinetics of ertapenem in healthy young volunteers. Amer College Clin Pharmacol 2001. Abstract 210E.

5.- Musson D, Majumdar A, Birk K et al. Pharmacokinetics of intramuscularly administered Ertapenem. Antimicrob Agents Chemother 2003; 47 (5): 1732-5.

6.- Laethem T, De Lepeleire I, Mc Crea J et al. Tissue penetration by ertapenem, a parenteral carbapenem administered once daily, in suction induced skin blister fluid in healthy young volunteers. Antimicrob Agents Chemother 2003; 47 (4): 1439-42.

7.- Fuchs P, Barry A, Brown S et al. In vitro activities of ertapenem (MK- 0826) against clinical bacterial isolates from 11 North American Medical Centers. Antimicrob Agents Chemother 2001; 45: 1915-8.

8.- Hoban D J, Palatnick N, Bellyou T et al. Comparative activity of MK-0826 (Ertapenem) against Canadian respiratory isolates Streptococcus pneumoniae and Haemophilus influenzae. Program and Abstracts of the 42th ICAAC San Diego, September 2002 Abstract E 1651.

9.- Aldrige K. Ertapenem (MK-0826), a new carbapenem: comparative in vitro activity against clinically significant anaerobes. Diag Microbiol Infect Dis 2002; (44): 181-6

10.- Livermore D, Oakton K J, Carter M W, Warner M. Activity of Ertapenem versus Enterobacteriaceae with potent $\beta$-lactamases. Antimicrob Agents Chemother 2001; 45: 2831-7.

11.- Paterson D L, Bonomo R A, Kolano J et al. Ertapenem resistance in extended spectrum betalactamase producing Klebsiella pneumoniae bloodstream isolates. 
Program and Abstracts of the 42th ICAAC San Diego, September 2002. Abstract C2-1886.

12.- Cercenado E, Cuevas O, García-Arias V, SánchezSomolinos M, Guinea J, Bouza E. Activity of ertapenem against recent clinical isolates of Enterobacteriaceae with potent betalactamases. Program and Abstracts of the 43th ICAAC Chicago, September 2003. Abstract E354A.

13.- Jacoby G, Chow N. Role of plasmid and chromosome in ertapenem resistance of Klebsiella pneumoniae. Program and Abstarcts of the 42th ICAAC, San Diego, September 2002. Abstract C1-1841.

14.- Silver L. Lack of apparent induction of betalactamase by ertapenem in Pseudomonas aeruginosa. Program and Abstracts of the 42th ICAAC, San Diego 2002. Abstract C1-1840.

15.- Friedland I R, Isaacs R, Mixson L et al. Use of surrogate antimicrobial agents to predict susceptibility to ertapenem. Diag Microbiol Infect Dis 2002; 43: $61-4$.

16.- Solomkin J, Yellin A, Rotstein O et al. Ertapenem versus piperacilina/tazobactam in the treatment of complicated intraabdominal infections. Ann Surg 2003; 237 (2): 235-45.

17.- Yellin A, Hasset J, Fernández A et al. Ertapenem monotherapy versus combination therapy with ceftriaxone plus metronidazole for treatment of complicated intraabdominal infections in adults. Intern J Antimi- crob Agents 2002; 20 (3): 165-73.

18.- Teppler H, Mc Carroll K, Gesser R, Woods G. Surgical infections with Enterococcus: outcome in patients treated with ertapenem versus piperacilin tazobactam. Surg Infect 2002; 3 (4): 337-49.

19.- Graham D, Lucasti C, Malafaia O et al. Ertapenem once daily versus piperacilina/tazobactam 4 times per day for treatment of complicated skin and skin structure infections in adults: results of a prospective, randomized, double blind multicenter study. Clin Infect Dis 2002; 34: 1460-8.

20.- Tomera K, Burdmann E, Pamo O et al. Ertapenem versus ceftriaxone followed by appropriate oral therapy for treatment of complicated urinary tract infections in adults: results of a prospective, randomized, double blind multicenter study. Antimicrob Agents Chemother 2002; 46: 2895-900.

21.- Ortiz-Ruiz G, Caballero-López J, Friedland J et al. A study evaluating the efficacy, safety and tolerability of ertapenem versus ceftriaxone for the treatment of community acquired pneumonia in adults. Clin Infect Dis 2002; 34: 1076-83.

22.- Roy S, Higareda I, Angel-Muller E et al. Protocol 023 study group. Results of a phase III randomized, doubleblind study of ertapenem (ETP) vs piperacillin/ tazobactam $(\mathrm{p} / \mathrm{t})$ for acute pelvic infection in women. Program and Abstracts of the 41th ICAAC Chicago, December 2001. Abstract 888.

Correspondencia a:

Ricardo Morales I.

E-mail: hamburgo@vtr.net 